\title{
The Hinges of Destiny: A Decision Analysis of Spectrum Users' Choices
}

\author{
Martin B.H. Weiss \\ School of Information Sciences \\ University of Pittsburgh \\ Pittsburgh, PA \\ mbw@pitt.edu
}

\author{
Liu Cui \\ School of Information Sciences \\ University of Pittsburgh \\ Pittsburgh, PA \\ lic49@pitt.edu
}

\begin{abstract}
Dynamic spectrum access (DSA) has been a technological approach that has received considerable research attention over the past decade. At the same time, the relative paucity of deployed systems using some of these technologies speaks to either a mismatch between research priorities and practical needs, regulatory immaturity, technology immaturity, or a combination of these. In this paper, we examine the business decision that a spectrum entrant must take with regard to technology choice. We use a simple decision-analytic framework using standard Net Present Value (NPV) calculations to analyze that decision. Our conclusion is that, using the rough cost estimates and a simple system model, that exclusive use offers a higher NPV than the alternatives. The second choice is cooperative sharing, followed by opportunistic sharing under optimistic spectrum availability and contention assumptions.
\end{abstract}

If our assumptions are relatively close to reality, it is therefore not surprising that we do not see greater adoption of DSA technologies - it is not the top choice for spectrum entrants with a long term view. The second choice, cooperative sharing, does occur, but in the form of MVNO agreements. The last option for a spectrum entrant is opportunistic sharing. Why should an entrant settle for the third best approach?

Keywords-Dynamic Spectrum Access, Cognitive Radio, Decision Analysis, Cost Analysis

\section{Choices are the Hinges of Destiny -- Pythagoras}

\section{INTRODUCTION}

At the $5^{\text {th }}$ IEEE International Symposium on Dynamic Spectrum Access Networks (DySPAN) in 2011, an undertone of the meeting was that academic research was irrelevant in practice. This did not show up in the papers in the published conference proceedings, but was clear in the questions and in the hallway conversations. Summarizing the main elements of the argument:

- after about a decade of research in DSA technologies, there has been little to show for the effort in the way of practical systems; and

- academic research has not been paying attention to priorities for commercial implementation.

This work was supported in part by US National Science Foundation Grant $\# 1149422$.
While these criticisms surely have some merit, there have been some notable contributions to this end (see, for example, [1-5]). In this paper, we do not seek to debate these observations, but rather to gain insight into the barriers to adoption of DSA techniques and technologies.

In its simplest form, spectrum sharing involves providing spectrum access opportunities to market or industry entrants whose application is best met by wireless systems. Spectrum access opportunities occur because license holders (i.e., spectrum incumbents) do not utilize their spectrum $100 \%$ of the time. Sharing can occur through cooperatively (i.e., through explicit bargaining) or opportunistically (i.e., without bargaining).

To address the criticisms described above means, in part, exploring the conditions under which DSA might be adopted. To do this, we must examine the incentives and constraints of both primary and secondary users. The incentives that primary users have for sharing have been studied (see [5] and others), though these studies often do not consider the business strategies, investment and technological risks that primary users face. Similarly, the decision processes of potential secondary users must be studied to understand the circumstances under which they adopt this technology. The choices confronted by potential secondary users in context has received little attention beyond the work of Tonmukayakul and Weiss [1] and Weiss [2].

We refer to potential secondary users in context because entrants have a range of choices they can make. In the simplest form (used by [1]) the entrant can choose to obtain a license, to use license-free spectrum or to use a secondary use technology. Within the domain of secondary use, they have a range of choices available (outlined very briefly above but described in more detail by [4]. Each of these choices carries risks, such as investment risks, technological risks, service risks, strategic risks as well as benefits, such as return on investment, QoS premiums, etc.

In this paper, we will build a first order model of the secondary user's decision across these choices, accounting in a simple way for these risks. Since the range of choices is large and highly context dependent we will focus only on spectrum entrants who intend to operate a direct infrastructure-based system in one of four ways (outlined below). In doing so, we do not include MVNO relationships, or the kinds of spectrum 
access that might occur under virtualized mobile networks [6]. We use Net Present Value (NPV) to demonstrate the investment and return for each spectrum choice, and summarize the preferred situations.

The paper is organized as follows: section II elaborates an entrant's spectrum choices and their consequences. Section III computes the NPV for based on a set of cost assumptions. Section IV examines the risks and potential management flexibility for each spectrum choice. Section V summarizes the paper and proposes future research.

\section{SPECTRUM CHOICES}

In our simplified choice regime, we model an infrastructure-based spectrum entrant who is confronted with four alternatives (summarized in Figure 1) - exclusive use, cooperative sharing, opportunistic sharing, and unlicensed usage. The spectrum entrant must make an irrevocable choice of one of these four approaches to provide a wireless service. We assume that the revenue that the entrant can obtain is not dependent on the approach that is chosen, but, for simplicity, is linearly dependent on the service quality s/he can achieve, the number of users reached, and the period of time that these can be reached. For simplicity, we assume the revenue is certain in all scenarios.

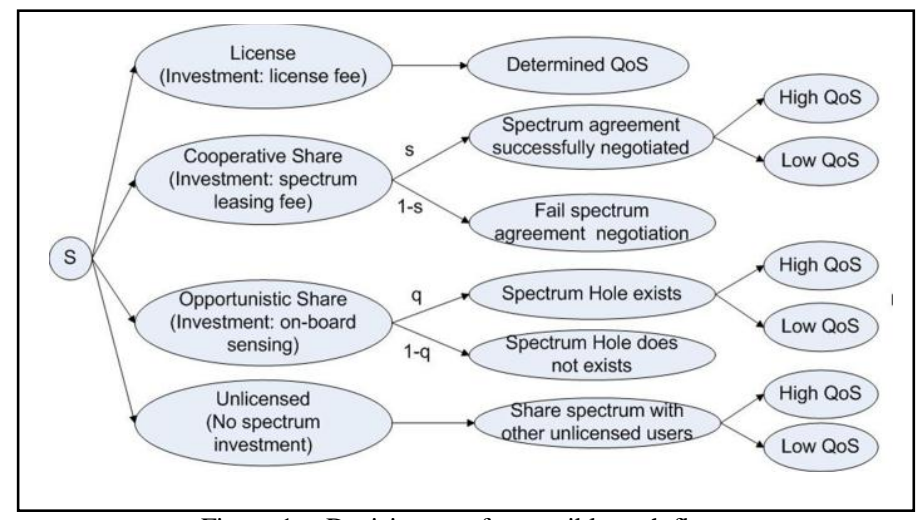

Figure 1. Decision tree for tangible cash flow

Before we delve into the details of the analysis, it is useful to consider these alternatives qualitatively (from [2]).

- Exclusive use requires that the entrant obtain a spectrum license via primary or secondary markets [7]. This gives the entrant optimal opportunity to engineer their systems to a desired quality level without involvement of other parties. The spectrum entrant becomes a license holder and primary user.

- Cooperative sharing means that spectrum entrants must first negotiate a spectrum use agreement with a license holder. We differentiate this from an MVNO in that we assume here that the entrant provides their own infrastructure. The spectrum entrant becomes a secondary user and can provide service subject to the priorities of the primary user. The service quality risk is somewhat higher because of that subordination; because it is a matter of contract, the quality depends more on the ability to enter into an agreement than it does on the execution of the agreement (assuming perfect remediation for contract breach). We assume also that the contract provides exclusivity for the secondary user.

- Opportunistic sharing means that spectrum entrants must have the capability to sense primary users' activities so they can take advantage of idle spectrum slots. There are numerous types of spectrum holes and context acquisition approaches (see [2, 3] for a discussion and analysis). The service quality risk under this approach is higher than under cooperative sharing for two reasons: (1) the spectrum entrant relies on the probability that spectrum holes are available at a sufficient frequency and bandwidth [3,8] and, (2) the spectrum holes may need to be shared with other opportunistic users, so the throughput of those holes is uncertain. For the purposes of this paper, we are implicitly assuming stochastic, exogenous spectrum holes, though this analysis could apply easily to other kinds of exogenous spectrum holes as well.

- Unlicensed means that all spectrum users have equal access to the bands (primary non-cooperative sharing according to [9]). Thus, the quality that any user can achieve depends on (1) the probability of other active unlicensed users at that time and (2) the characteristics of the MAC protocol in use. However, there is no cost for spectrum licenses, contracts or sensing required in this approach, so it may well be the cheapest.

For our more detailed analysis below, we assume:

- Suitable cooperative sharing agreements can be negotiated with a probability $s$, and that these agreements provide exclusive use for the spectrum entrant for the duration of the contract. We assume that the agreement is for a fraction $e$ of the license holder's electrospace. We also assume that these do not require spectrum entrants to sense the spectrum. Formally, $0<s \leq 1$ and $0<e \leq 1$.

- Opportunistic sharing uses cognitive radios with onboard sensing. We assume that an adequate spectrum hole is available with some probability. We also assume that $n$ other users share the spectrum hole with a probability $u(n)$, and that each spectrum user has an equal share of the spectrum hole's capacity. To simplify the analysis, we assume that the spectrum hole has sufficient bandwidth with a probability $q$, where $q$ is the probability that the spectrum hole is adequate after sharing it with the $n$ users who are present. Formally, $0<q \leq 1$

- $\quad$ There is enough demand, so revenue only depends on the quality and net service provision. We will consider the impact of this assumption in section III-D.

- $\quad$ Primary users establish infrastructure and purchase a spectrum license at the beginning of the project and make an irrevocable commitment for the lifespan of the project. We will revisit this assumption in section IV, where we consider managerial flexibility. 


\section{Net PResent VAlue Analysis}

\section{A. Investment}

From the discussion above, it is clear that each spectrum choice that a spectrum entrant can make involves a different investment as well as a different return on that investment. As summarized in Table 1, each method incurs an infrastructure cost and maintenance (IM) fee which can vary across methods. In addition to these costs, primary users must pay the upfront spectrum license fee, cooperative secondary users must pay a periodic spectrum leasing fee as long as they operate, and opportunistic secondary users have to invest in sensing before providing services (see [3]).

\section{B. Benefit}

Our consideration of benefit is focused on the achievable revenues. Specifically, we frame revenue as a function of the quality, duration and reach of the service ${ }^{1}$. Here, we let the quality of service (QoS) of a wireless service depends on the absolute throughput as well as its variation. The duration of service is the amount of time a service is available over the study interval. Finally, the reach of the service is a combination of the overall coverage area as well as the population density of that area.

TABLE I. COST AND BENEFIT FOR EACH CASE

\begin{tabular}{|c|c|c|}
\hline & Cost & Benefit \\
\hline $\begin{array}{l}\text { Exclusive } \\
\text { Usage }\end{array}$ & $\begin{array}{l}\text { Infrastructure, IM, } \\
\text { Spectrum license fee }\end{array}$ & $\begin{array}{l}\text { High and deterministic QoS, High } \\
\text { population density }\end{array}$ \\
\hline $\begin{array}{l}\text { Coop } \\
\text { Sharing }\end{array}$ & $\begin{array}{l}\text { Infrastructure, IM, } \\
\text { Spectrum leasing fee }\end{array}$ & $\begin{array}{l}\text { s-enough bandwidth } \\
\text { s depends on market } \\
\text { High population density }\end{array}$ \\
\hline Op Sharing & $\begin{array}{l}\text { Infrastructure, IM, } \\
\text { Sensing function }\end{array}$ & $\begin{array}{l}\text { q-enough bandwidth } \\
\text { q depends on PU behavior, sensing } \\
\text { performance, number of users }\end{array}$ \\
\hline $\begin{array}{l}\text { Unlicensed } \\
\text { Usage }\end{array}$ & Infrastructure, IM & $\begin{array}{l}\gamma \text {-enough bandwidth } \\
\gamma \text { depends on competition (number } \\
\text { of users) } \\
\text { High population density }\end{array}$ \\
\hline
\end{tabular}

Because of the exclusivity that obtains to spectrum licensees, we assume that primary users have the highest and most predictable QoS. The duration and reach of the service is explicitly priced into the cost of the license and is under the control of the licensee. The question that exclusive users must evaluate is whether they can gain an adequate return on the investment of the spectrum license.

Users who engage in cooperative sharing negotiate a spectrum lease with primary users. In bilateral negotiations, sharing can take on a number of forms (See [10] for an exposition on spectrum sharing negotiations in early US broadcast radio). For the sake of discussion, let us consider a few possibilities:

1. A contract that leases a spectrum band continuously over a longer investment horizon (i.e., months/years) for a subset of the license service area;

\footnotetext{
${ }^{1}$ We ignore any strategic benefits for the purpose of this paper.
}

2. A contract that provides for defined periodic access (e.g., fixed hours or days) over the investment horizon for the entire license service area;

3. A contract that leases a subset of the spectrum for continuously over a longer investment horizon (i.e., months/years) for the entire license service area;

4. A contract that provides for defined periodic access (e.g., fixed hours or days) over the investment horizon for the entire license service area for a subset of the spectrum license

Clearly, many additional variations are possible. The point in describing a few possibilities is that, in general, we can assume that the revenue is lower for cooperative secondary users than for exclusive users either because of the quality provided, the (average) duration of the access or the reach of the access.

Spectrum entrants who choose opportunistic sharing will, in general, find that their revenue is subject to greater uncertainty because quality, duration and reach are all random variables in practice (depending on the type of spectrum hole). Thus, they may find that their revenues are sometimes higher than cooperative sharers but not always. The sources of uncertainty are the frequency, size and bandwidth of the spectrum holes as well as the likelihood of other opportunistic users who want to share the spectrum hole. Thus, entrants must determine whether the spectrum hole density is sufficient (and in the right places) to warrant the investment in infrastructure and sensing that is necessary for this approach to work [2]. Finally, spectrum entrants who choose to operate in unlicensed bands are, in general, subject to greater interference from other unlicensed users and can usually operate systems of limited reach unless they invest in many more access points.

As we mentioned above, we equate benefit with revenue for the purpose of this paper, and that this is a linear function of quality, duration and reach with complete certainty over the investment horizon. For our first order analysis, we use a simple multiplicative form of this function:

$$
R=\mu \cdot \delta \cdot t \cdot \beta \cdot a
$$

where, $\mu \in[0,1]$ indicates the external impact on QoS level, calibrated such that a license holders' QoS level $=1, \mu$ equals to $s$ for cooperative sharing, which is the probability of successful negotiated a spectrum contract. $\mu$ is the probability of spectrum hole availability for opportunistic spectrum users. $\delta \in[0,1]$ indicates the QoS variance (similar as jitter if the QoS is measured as delay). In this paper, we consider $\delta$ as contention rate. $\delta \rightarrow 1$ means the contention rate is negligible. In a real system, contention increases costs for retransmission and requires a larger buffer to smooth the jitter. We only consider the impact from QoS variance on revenue in this paper. $t \in[0,1]$ represents the operation time, again with respect to a license holder. $\beta \in[0,1]$ specifies the coverage with $\beta=1$ means the wireless service reaches the entire geographic area listed in the spectrum license. Finally, $a$ is a constant and represents the revenue per unit of service delivered. Since we are interested in comparing the decision of a spectrum entrant, we assume this is constant for all modes of spectrum access. 


\section{NPV for the Four Choices}

In this section we use Net Present Value (NPV) to analyze the four spectrum choices. The NPV is an indicator of the valuation of each alternative. With a particular project, if the NPV is positive, the project brings revenue to the firm. If the NPV is negative, the project subtracts value from the firm. If the NPV equals to zero, the project does not bring monetary value to the firm. The formula of calculating NPV is

$$
N P V=\sum_{t=0}^{N} \frac{N C F_{t}}{(1+i)^{t}}
$$

where, $N C F_{t}$ is the net cash flow at time $t, i$ is the annual riskfree interest rate, $N$ is the total number of period.

1) Exclusive use: We assume that spectrum users need to provide the wireless service for the entire service area listed in the spectrum license (for economic and regulatory reasons). For simplicity, we assume that 10 base stations are needed to cover the entire region with a cost of $\$ 100,000$ per station. For the sake of reliability, footprints have overlap. Thus, we assume that with 10 base stations, they cover $30,000,000 \mathrm{~m}^{2}$ (radius $=1000 \mathrm{~m}$ per station). Included in the $\$ 100,000$ expense is $\$ 25,000$ for the cost of the radio radio, and $\$ 75,000$ for the construction cost of the base station. The spectrum license fee and infrastructure cost are incurred up front. We assume that with a $\$ 10$ million spectrum license, the spectrum entrant can operation full time $(t=1)$ and maximize reach $(\beta=1)$. Since it is exclusive usage, primary users have enough bandwdith $(\mu=1)$ and the variance is negligible $(\delta=1)$. The maintanence cost is $10 \%$ of the infrastructure cost for all cases. The backhaul cost is assumed to be $\$ 150$ per month per base station for exclusive use and for the two flavors of sharing.

$$
N P V_{P U}=-I_{P U}-C_{P U}+\sum_{t=1}^{N} \frac{R_{P U}-I M}{(1+i)^{t}}
$$

Where, $I_{P U}$ is the infrastructure cost for exclusive usage, $C_{P U}$ is the spectrum license fee, $R_{P U}$ is the annual revenue for exclusive usage, $I M$ is the maintanence cost plus backhaul charge per year.

2) Cooperative Sharing: In this approach, the spectrum entrant incurs no upfront spectrum license fee and the infrastructure cost is the same as exclusive usage if spectrum user plans to provide wireless service to the entire region. We assume that the infrastructure cost decreases with the geographic coverage linearly for all cases. While the leasing contract can take many forms, for the purpose of this analysis, we assume that the spectrum leasing fee depends on the operating time, reach, and QoS level, and occurs annually. We set the annual cost for spectrum leasing when $t=\delta=\beta=1$ to be $\$ 1$ million $^{2}$. In all cases, we set the probability $s$ (which describes the probability that spectrum sharing agreement can be negotiated) to 0.8 . As we assumed before, the QoS for cooperative secondary users is the same as exclusive usage

\footnotetext{
2 At this price, the lease is $150 \%$ (non-discounted) of the license cost over a 15 year lease. In reality, the price for the lease is dependent not only on the cost of a spectrum license, but also on the expected revenues an operator could receive.
}

after they successfully negotiated the spectrum agreement, thus $\delta=1$.

$$
N P V_{C S}=-I_{C S}+\sum_{t=1}^{N} \frac{R_{C S}-I M-C_{S L}}{(1+i)^{t}}
$$

Where, $I_{C S}$ is the infrastructure cost for cooperative sharing, $R_{C S}$ is the annual revenue, $C_{S L}$ is the spectrum leasing fee. The QoS for cooperative sharing depends on the spectrum investment. We assume that spectrum cost increases with contract term. With higher cost they get better QoS. We use two cases depicting different spectrum leasing contracts. Case 1 refers to situation when users invest more money in spectrum resulting in a $\operatorname{QoS}(t=\delta=\beta=1)$. In case 2 , users invest less, which results in a worse $\operatorname{QoS}(t=0.6, \delta=1, \beta=0.5)$.

3) Opportunistic sharing: This approach requires equipment that can operate over a larger frequency range as well as requiring a sensing function. To simplify the analsyis, we assume that sensing will be performed by an external sensor network (following [11]), and we assume that they need 10 sensors with cost of $\$ 10,000 \mathrm{each}^{3}$ (this frees us from having to estimate the number of users, as would be necessary in the cognitive radio case). We assume that the radio cost is $25 \%$ more expensive than the previous two approaches, which is $\$ 31,250$. Therefore, the total cost for opportunistis sharing is $\$ 1,162,500$.

In comparison with the cooperative sharing approach, opportunitsitic users do not have much control over QoS. The QoS in opportunistic sharing is impacted by the spectrum availability and contention probability. The former is the result of the primary users' usage and the latter is determined by other opportunistic users' operations. While much is unknown about spectrum hole density, researchers (e.g. [8]) have built some preliminary models using Markov and Semi-Markov chains. Considerably more work is needed in this arena to characterize and model real-world spectrum holes, cince the characteristics of spectrum holes have a significant impact [3].

We continue with two cases to illustrate opportunistic sharing. In the first case the number of opportunistic users is low (maximum 2) and the spectrum hole availibility is high $(0.5)$, it leads to higher QoS, $\beta=1$ in this case. In the second scenario, the number of opporunistic users is high (maximum $5)$ and the spectrum hole availibility is low (0.3), so the QoS is low, $\beta=0.5$. We further assume that opportunistic users work in the TDMA mode and that the number of users is uniformly distributed, thus, $t=E(u(n) \cdot(1 / n)$. The contention rate depends on the number of users; we assume that the contention rate for two cases are 0.8 and 0.7 , repectively. The NPV of opportunistic sharing is thus computed as:

$$
N P V_{O S}=-I_{O S}+\sum_{t=1}^{N} \frac{R_{P U}-I M}{(1+i)^{t}}
$$

where $I_{O S}$ is the infrastructure cost for opportunistic sharing, $R_{P U}$ is the annual revenue.

\footnotetext{
3 This is an approximate price of an RFEye spectrum monitor (http://j.mp/JqHNGD) .
} 
4) Unlicensed usage: Spectrum entrants have the least infrastructure cost per station in this case because the equipment is heavily standardized and the transmit power is limited. At the same time, the reach for each station is restricted (cell radius $=100 \mathrm{~m}$ ) because larger cells result in higher congestion. Thus, it requires 950 tranmitters to cover the entire region. We assume that each micro cell only costs $\$ 600$, where $\$ 500$ is the cost for radio and $\$ 100$ is the cost for installation. This results in a total infrastructure cost of $\$ 570,000$. The backhaul cost is assumed to be $\$ 50$ per month (similar to DSL) per site, although this could be reduced by careful attention to the access network architecture. For simplicity, we assume that unlicensed users work under TDMA, and they get desired QoS whenever it is transmitted withouth contention. We also assume that there is no external factor impacting QoS other than MAC protocol and contention, therefore, $\mu=1$. In contrast to opportunistic or cooperative users, unlicensed users can work where ever they want, so $\beta=1$. The transmission time $(t)$ depends on the number of active unlicensed users. As described above, $n$ users share the spectrum with probability $u(n)$. Like oppotunistic sharing, unlicensed users' QoS is determined by other users' behaviors. If they are lucky enough, they are the only user and operate as though usage was exclusive. If there are other unlicensed users, the contention rate and the workable portion is low. We also deploy two cases in unlicensed bands. In the first one, $n \in[1,20]$ with uniform distribution. Thus, $t=E(u(n) \cdot(1 / n))$, and contetion rate is $0.6, \beta=1$. In the second case, $n \in[1,50]$. Due to the large number of unlicensed users and non-perfect coordination, the contension is prone to happen $(\varphi=0.5), \beta=1$. We calculate the NPV for unlicensed users as follows:

$$
N P V_{U}=-I_{U}+\sum_{t=1}^{N} \frac{R_{U}-I M}{(1+i)^{t}}
$$

where, $I_{U}$ is the infrastructure cost for unlicensed users and $R_{U}$ is the annual revenue.

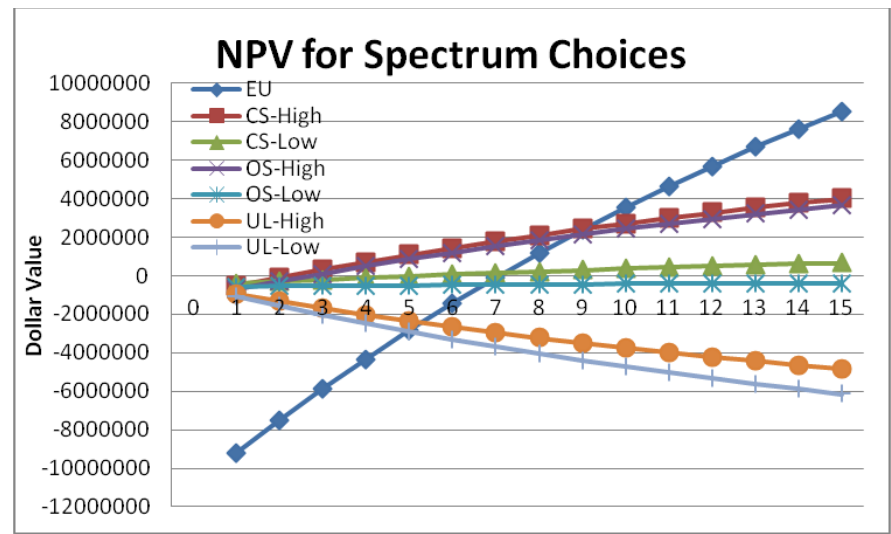

Figure 2. NPV for Sepctrum Choices

Figure 2 illustrates the NPV for each of the 15 years of the project life for each scenario. Thus, it provides us with project NPV (in the $15^{\text {th }}$ year) as well as the breakeven point (when it crosses NPV $=0$ ) using the assumptions described above. We see that exclusive use has the largest return in long-term, breaking even in the $8^{\text {th }}$ year. Cooperative sharing for high QoS has the second highest long-term NPV, though marginally so, being $\$ 0.3$ million more than high QoS opportunistic sharing. It is important to reiterate here that the spectrum entrant cannot control whether a high outcome is possible, especially in the opportunistic case, because it depends on the uncertain availability of spectrum holes and the uncertain number of other users using those holes. In the cooperative case, there is also uncertainty in outcome, but it can be, to some extent, influenced by the entrant (i.e., it is endogenous [2]) based on the leasing price $\mathrm{s} / \mathrm{he}$ is willing to pay. Unlicensed service is never profitable under our assumptions because of the high backhaul costs and is consistent with other research studies [12, 13]. More nuanced conclusions that might be drawn include:

- If spectrum users need long-term maximum gain, and they have enough capital for infrastructure and a spectrum license (assuming one is available), being an exclusive user is the preferred choice.

- If the spectrum user wants to explore a new wireless services, the best choice is unlicensed but over a limited area (to control backhaul costs), since no agreement is needed and spectrum is always available through QoS is variable.

- If spectrum users do not have enough capital at the beginning, but have stable annual funding for the project, cooperative spectrum sharing is the best choice, since it leads to high revenue with relative less sunk cost.

- If spectrum users have some capital at the beginning of the project but unpredictable ongoing funding, opportunistic sharing may be preferred, since the NPV is positive and the annual cost is limited to backhaul and maintenance, which would have to be incurred anyway.

\section{Sensitivity Analysis}

Clearly, the outcomes reported above are dependent on the assumptions made in this paper. To address this, we will examine the cost levels that will cause these outcomes to change. For example, we will answer question like: at what spectrum license fee does exclusive usage lead to the same long-term revenue as cooperative sharing?

1) Exclusive Usage: According to above assumptions, if the probability of successfully negotiation is 0.8 , the long-term NPV for high QoS cooperative sharing is $\$ 4,171,145$. Under the condition that spectrum users will cover the entire license region, when the spectrum license fee for exclusive usage becomes $\$ 14,531,521$, the NPV for exclusive usage is the same as cooperative sharing (a $45 \%$ increase). Therefore, if spectrum license is more expensive than this value, spectrum users would choose cooperative sharing over exclusive use. Outside the model, there are also other considerations in spectrum choices, such as managerial flexibility (in section IV) and cash availibility. If the company has a large amount of cash available at the beginning of operation and is not certain about making an annual investment, exclusive usage may still 
be a better choice even though the long-term NPV may be less than high quality cooperative sharing.

2) Cooperative Sharing: Two parameters impact cooperative sharing. The first one is the likelihood of successful negotiation $(s)$, and the second one is the spectrum leasing price. Cooperative sharing is very sensitive to $s$. When $s=0.784047$ (1.6\% less than original assumption) or the unit spectrum leasing charge is equal to $\$ 1,031,906$, the high cooperative sharing leads to the same NPV as high quality opportunistic sharing (assuming this outcome can be obtained). Another consideration (outside the model) for cooperative sharing is changing to exclusive use. They have the same infrastructure cost and the only difference is their spectrum investment. If the cooperative user is certain about business model and a spectrum license is available, s/he can change to exclusive usage without wasting their previous investment.

3) Opportunistic Sharing: In this strategy, users face uncertainties that they cannot control. The first uncertainty is spectrum hole availibility, the second one is the number of opportunistic users, and the third one is sensing cost. (1) The spectrum hole availability is a result of a primary users' spectrum usage pattern. If the spectrum hole availibility is $11.5 \%$, even if there is only one opportunistic user and the spectrum hole covers the entire region, it leads to a negative NPV. (2) If the number of other opportunistic users increase from 2 to 10 , even if the spectrum hole is available all the time, the NPV nearly equals high QoS unlicensed usage. (3) Recall that we assume that the cost for the sensing capability is $\$ 10 \mathrm{~K}$ each, with one sensor per base station. When the sensing cost reduces to $\$ 1 \mathrm{~K}$ per base station, high opportunistic sharing produces a higher NPV than low cooperative sharing and it is nearly equal to high cooperative sharing. Outside the model, when opportunistic sharing is not profitable, spectrum users can change to cooperative sharing. Changing to cooperative sharing requries a spectrum leasing fee and provides relative certainty of QoS. The alternative, changing to unlicensed usage is not profitable.

Another key point for spectrum entrants to consider before undertaking opportunistic sharing is that primary users tend to maximize their profits. In other words, primary users will operate as long as it is profitable to do so. Therefore, the spectrum that left for opportunistic sharing may not be as valuable as in exclusive and cooperative sharing. For example, the spectrum hole may exist in area with a smaller population or during a time with less active customers. Furthermore, license holders may behave opportunistically [2], which could significantly affect the expected spectrum hole availability statistics (such as those calculated in [8]).

4) Unlicensed: Like opportunistic sharing, unlicensed users face uncertainty of number of users in the same band over the same area. The results of our analysis are due to the larger number of access points needed and the necessity of providing backhaul to each. Costs can be reduced if fewer access points are needed, but this may run afoul of transmit power standards in the band and will certainly increase the likelihood that competing users will exist, which drives down QoS. Thus, we vary only backhaul costs, not access point density. The upper unlicensed choice outcome becomes profitable when backhaul costs are $\$ 9$ (or $18 \%$ of the estimated value). Note that the outcome is also dependent on the number of other users. We assume that 37 is the maximum number of unlicensed users that the spectrum resource can support.

5) General parameters: Two general parameters have a large influence on the spectrum choice-coverage and unit revenue. Coverage determines the infrastructure cost, the infrastructure cost increases with the size of the footprint due to the physical characteristics of electromagnetic waves. The unit revenue reflects the demand from customer side. Spectrum users have a clearer view after they know the demand. In general, varying the coverage (or reach) parameter does not alter that outcome

Performing sensitivity analysis on these the unit revenue parameter poses challenges beyond the scope of this paper. We assumed that the revenue is linear with QoS for simplicity. However, in reality the revenue may be log normal with QoS. That is the revenue is very sensitive to QoS when it is relatively low. In addition, spectrum users may have different business and service models which also impact revenues. Finally, the license fee negotiated in the cooperative case is will likely be dependent on the expected revenues, resulting in the need for a model that dynamically adapts.

\section{MANAGERIAL FLEXIBILITY}

Tangible cash-flow is only one aspect that a firm must consider. Another important aspect that does not show in the NPV calculated above managerial flexibility. Having the flexibility to deal with uncertainties has value. The real options approach is popular for investigating risks and flexibility in a way that is difficult to capture in NPV-based models [14]. The rationale for using real options is that investments always lead to uncertainties in the future. Real option gives the firm the right but not obligation to take a specific action in the future. Applying real options to the secondary user decision problem will be treated explicitly in a future paper, but we would like to point out some of the benefits of this approach here.

\section{A. Risk and Benefit}

According to [15], a complete investment lifecycle can be divided into six stages. Every investment begins from inception stage, where investment is an implicit opportunity. At the recognition stage, the investment is deemed a practical opportunity. After making the investment decision, the project enters the building stage. The flag for operation stage is that the project starts having direct and measurable payoffs. In the retirement stage, indirect payoffs come from the vast investment in technological assets and its capabilities. When these assets and capabilities no longer produce payoffs, the investment reaches the obsolescence stage. 
TABLE II -- RISKS AND OPTIONS IN THE INVESTMENT LIFE CYCLE

\begin{tabular}{|c|c|c|c|c|c|c|c|c|c|c|c|}
\hline \multicolumn{2}{|r|}{ Investment Life Cycle } & \multirow{2}{*}{\multicolumn{5}{|c|}{ 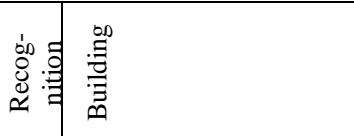 }} & \multicolumn{5}{|c|}{ Operation } \\
\hline & & & & & & & \multirow{2}{*}{ 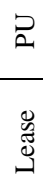 } & \multirow{2}{*}{$\frac{0}{0}$} & \multirow{2}{*}{$\frac{\widetilde{0}}{\frac{0}{3}}$} & \multirow{2}{*}{ 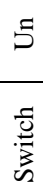 } & \multirow{2}{*}{ 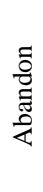 } \\
\hline Risk & & $\frac{\grave{d}}{\stackrel{0}{\Delta}}$ & 芴司 & $\begin{array}{l}\mathscr{U} \\
0 \\
0 \\
ن\end{array}$ & $\begin{array}{l}\mathscr{\delta} \\
\text { on. } \\
\text { ô. }\end{array}$ & $\stackrel{\mathscr{Z}}{\underline{\Xi}}$ & & & & & \\
\hline \multirow[t]{3}{*}{ Monetary } & M1-firm cannot afford the project & + & & + & & + & + & + & + & & + \\
\hline & M2 - expected costs are not in line with projected benefits & + & & & & & + & + & + & & + \\
\hline & M3-shared spectrum is very expensive & & & & + & + & & + & & & + \\
\hline Competition & $\mathrm{C} 1$ - competition for resource is high & & + & + & & & & & + & + & \\
\hline \multirow[t]{3}{*}{ Environmental } & E1-action of regulatory body & + & & & & & & + & + & + & + \\
\hline & E2-spectrum market liquidity & & + & & + & + & & + & & & \\
\hline & E3-spectrum license is not available & + & & + & + & + & & & & & \\
\hline \multirow[t]{3}{*}{ Technological } & T1-system may be infeasible with current resource & & + & + & & & & + & + & + & + \\
\hline & T2 - spectrum environment changes system requirements & & + & + & & & & + & + & + & \\
\hline & T3-Interference from other system is high & & + & + & & & & & + & + & \\
\hline
\end{tabular}

Although risks exist in the entire lifecycle of investment, we only focus here on the three stages that involve a large investment and high risks. They are the recognition, building, and operation stages. At the recognition stage, companies gather information and make estimates about costs, benefits, and risks. In the building stage, companies make needed investments (e.g., transmitters, base stations, etc.) and establish networks. They also need to make their spectrum access decision in the building stage. They have four choices as illustrated in Section IIA. Different spectrum decisions in the building stages lead to different risks in the operations stage, where spectrum users start providing wireless services to customers and earn revenues based on the investment in infrastructure and spectrum.

Risks in these three stages fall into four generic categories.

- Monetary risk is due to the uncertainty about the firm's ability to complete a project with long-term benefits. For simplicity, we do not consider demand for this paper. However, it is possible that spectrum users cannot cover their investments in infrastructure and spectrum if demand is weaker than anticipated.

- Competition risk comes from the responses of other firms in the industry due to entry. For example, if we consider the spectrum access dimension, we see that incumbents do not face competition risks due to spectrum access, since their spectrum license is exclusive. Cooperative spectrum sharing only encounters competition when they lease spectrum from license holders. They do not compete with others during after making the deal. Opportunistic spectrum sharing and spectrum users in unlicensed bands confront competition all the time and there is no guarantee of successful operation.

- Environmental risk considers two aspects. The first one is the regulation environment, which includes the FCC's regulation on spectrum assignment and allocation. For example, the FCC may open more unlicensed bands to stimulate innovation. It may also auction more spectrum licensees to boost competitions.
The second aspect is the market environment. Although the spectrum may be traded in the private market [16], it is still generally not considered a liquid market [7]. There is no guarantee that the transaction cost will be low or there will be enough cooperative spectrum sharing for secondary users.

- Technological risks come mainly from possible changes in the spectrum environment. For example, the existing infrastructure and services may not be compatible for available frequency bands. The physical characteristics of electromagnetic waves determine the requirements of infrastructure and possible services. Interference brought by other wireless systems that transmit in adjacent bands and geographic areas is another major technological risk.

This risk list is by no means exhaustive. There are other risks such as (1) project risks which consider project scope and staff's ability to implement the project; (2) functionality risks which related to the project design; and (3) organizational risks which reflect firm's cooperation and adoption of the project.

\section{B. Flexibility in spectrum utilization.}

1) Defer: is the ability to postpone the investment to learn more about the potential risks and outcomes of the project, and adjust to the varying situations. The defer option is available for all four choices in the recognition stage. Moreover, spectrum users can delay establishing infrastructure after they buy the spectrum license from the FCC. The maximum delay is 5 years. Other spectrum users do not have delay options in building stage.

2) Switch: in this project, spectrum users have the flexbility to change spectrum choices, except for primary users. For example, if the cooperative spectrum user finds the spectrum market is not liquid or the price for shared bands is too high, he can swtich to opportunistic spectrum sharing by extablishing sensing technology or unlicensed bands by no extra expense. Unlicensed users can switch to secondary users if the resource competition drives the QoS to be unacceptable. However, primary users do not have the ability to switch.

3) Lease: when the project payoff is too low, the resource (spectrum) can be leased. Only primary users can lease 
spectrum, since we assume that cooperative secondary users consume all shared bands they purchased.

4) Abandon: Project will be abandoned if the project payoff cannot cover the cost. In reality, the alternative is stopresume, where the project is terminated and put the available asset to other valuable projects. This option is outside the scope of this paper.

\section{Mapping risks and options in each case}

1) Exclusive Usage: As shown in Table II, if the company chooses to be a primary user in the building stage, they need to purchase the FCC issued spectrum license. the most possible risks in the operation stage are monetary related and leasing spectrum is a shadow option for risks. All other spectrum choices lead to more risks (uncertainties) than primary users in operation stage. As required for the spectrum license, primary users have to provide wireless services within 5 years from purchase. During these 5 years, they can lease spectrum to others. This action provides two benefits: they earn monetary compensation and they can better assess supply and demand and therefore avoid potential risks of losing money in novel services. In sum, primary users actually apply lease and defer together to manage risks. We use NPV to analyze an extreme case, in which primary users lease their entire spectrum for five years and then build their own infrasturcutre (Figure 3). We use the same cost and revenue assumptions as above and assume that the spectrum leasing fee is $\$ 1.5$ million per year.

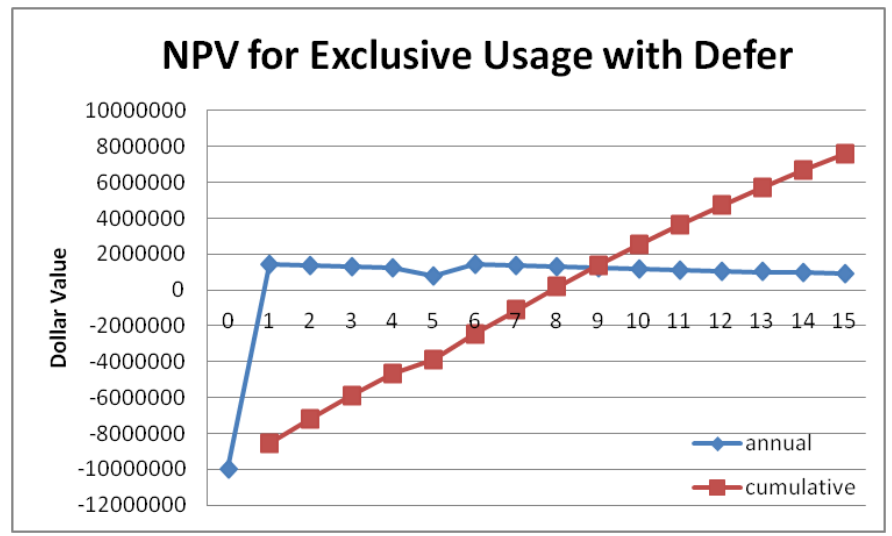

Figure 3. Exclusive usage NPV with Defer

2) Cooperative sharing: Cooperative users confront monetary risks that come from both infrastructure and spectrum. They also face potential competition from license holders when leasing spectrum, and regulatory action; finally, spectrum markets highly affect cooperative secondary users. Technological risks are due to the uncertainty of spectrum supply. Cooperative secondary users may encounter challenges if the radios they invested in are designed for frequency bands that are not available. The option for cooperative secondary users is switch, which means they can change their spectrum usage choice. For example, if they have enough capital to invest in a license when one is available and they have a profitable project, cooperative secondary users can become license holders (exclusive users) to manage regulation, technology and competition risks.

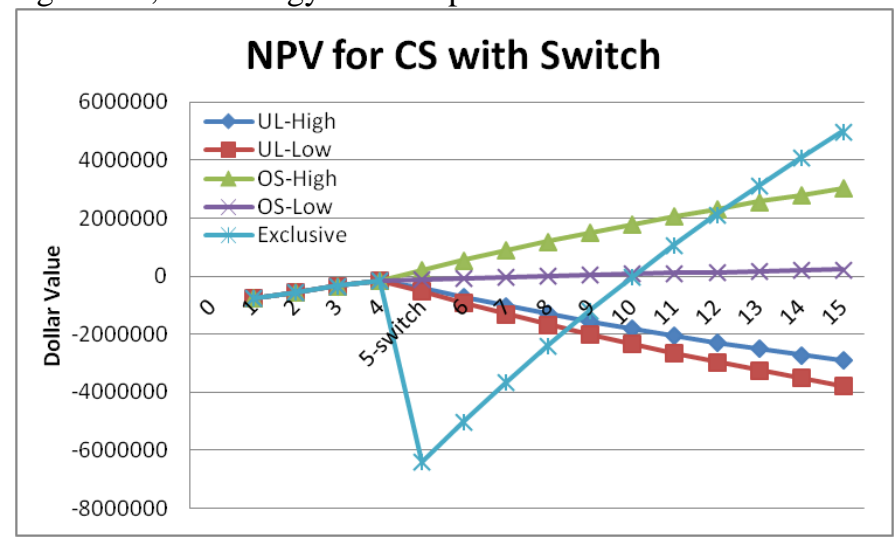

Figure 4. NPV for Cooperative Shairng with Switch Option

For this calculation, we assume that the spectrum user is currently in the low cooperative sharing state, and that the spectrum market is not feasible in year 4 but that they can change to another spectrum choice in year 5. Further, we assume that from this point all spectrum choices are based on full coverage. In Figure 4, we show the NPV for the switching option. All sharing methods lead to positive NPV (but not unlicensed). Exclusive usage wins due to its QoS guarantee, but it requires that the firm have enough cash for the license and that the license is available. There may also be a fee for changing bands.

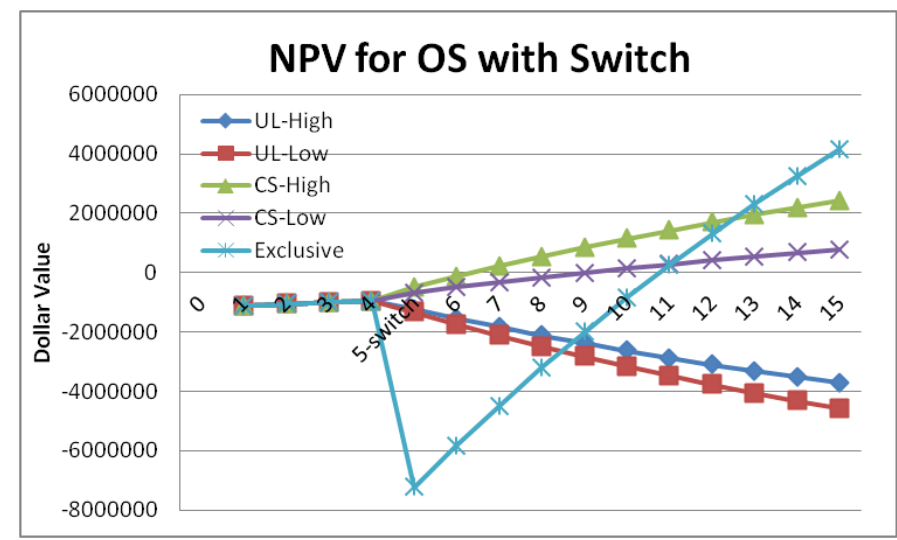

Figure 5. NPV for Opportunistic Sharing with Switch Option

3) Opportunistic Sharing If opportunistic sharing is of high quality (due to low contention and high spectrum hole availability), it produces the second highest NPV. However, to adopt this approach, the firm needs to upgrade its radios to those that can accommodate larger frequency bands and include sensing. When cooperative users switch to the unlicensed band, they need to rebuild the entire base station network. Due to the infrastructure reestablishment and high backhaul charge, unlicensed usage is unprofitable. 


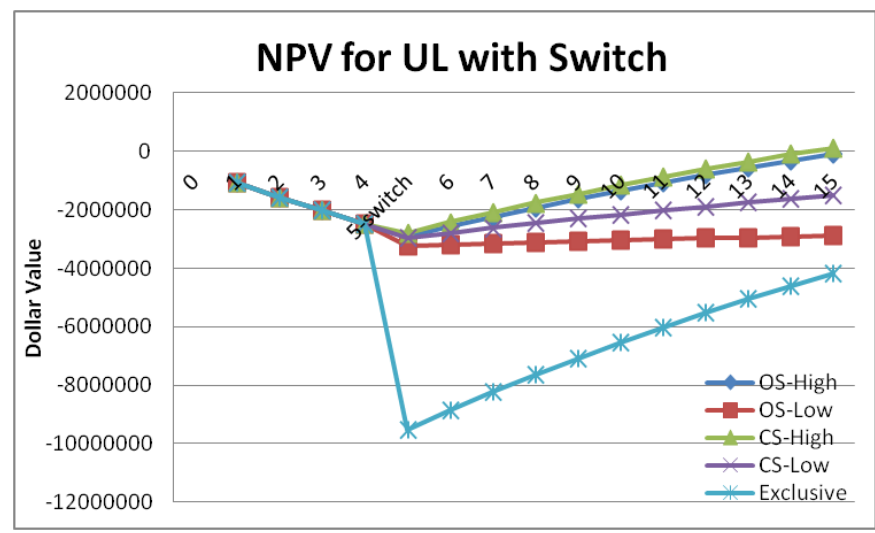

Figure 6. NPV for Unlicensed Usage with Switch Option

4) Unlicensed users: have the most intense competition for access and the interference is unpredictable. Therefore, there is no guarantee of QoS. Unlicensed users can be seen as an explore option, since they have the least sunk cost and the most flexibility in spectrum choices. As above, we assume that unlicensed usage is under low quality at stage 4 and confront spectrum choice at year 5. Figure 6 depicts the revenue for unlicensed users changing to other options. Not of all methods lead to positive revenue due to the sunk investment in unlicensed infrastructure and backhaul charge. However, if we extends the operation time to 20 years, exclusvie and cooperative sharing become profitable.

\section{CONCLUSIONS AND FUTURE RESEARCH}

This paper was a continuation of our research into secondary users and secondary user behavior. We have built a simple decision model to examine the choices that a potential spectrum entrant might make, and have developed this with some very rough estimates of some of the key cost and revenue components. Given the numbers that we used, we see that exclusive use produces the highest NPV, though it is clear that this could change as our model becomes more sophisticated and our cost estimates more accurate. The second best choice is cooperative sharing. This is because QoS is predictable in this outcome, even if the success at negotiation is not. Opportunistic sharing ends up being the third choice, mostly because of the added uncertainty. Finally, unlicensed sharing is generally not profitable for large scale deployments like this largely because of the high backhaul costs; these would have to be quite cheap indeed in order for this approach to become more attractive to spectrum entrants.

Understanding and managing the risks faced by spectrum entrants has received little attention ([17] is one of the few examples). We plan on applying real options analysis to this problem to better understand and model the problem(s) faced by secondary users with the ultimate goal of supporting the adoption of DSA technologies in practice. Doing this in a realistic way requires that we first develop a more sophisticated decision model that is supported by better technical models. It is also necessary to better calibrate the cost and revenue estimates so that the outcomes are more closely aligned with current or projected reality. A more sophisticated analysis of the risks faced by a spectrum entrant will be addressed using real options. Finally, we plan on incorporating these models into a an agent-based computational economics model (similar to [1]) to provide more sophisticated insight into the choice tradeoffs faced by secondary users.

\section{REFERENCES}

[1] A. Tonmukayakul and M. B. H. Weiss, "A study of secondary spectrum use using agent-based compuational economics," Netnomics, vol. 9, pp. 125-151, 2008.

[2] M. B. H. Weiss, "Spatio-Temporal Spectrum Holes and the Secondary User," in IEEE Dynamic Spectrum Access Networks, Aachen Germany, 2011.

[3] M. B. H. Weiss, et al., "Spatio-Temporal spectrum modelling: Taxonomy and economic evaluation of context acquisition," Telecommunications Policy, vol. 36, pp. 335-348, 20122012.

[4] J. Peha, "Sharing Spectrum Through Spectrum Policy Reform and Cognitive Radio," Proceedings of the IEEE, vol. 97, 2009.

[5] J. M. Peha and S. Panichpapiboon, "Real-time secondary markets for spectrum," Telecommunications Policy, vol. 28, pp. 603-618, 2004.

[6] T. K. Forde and L. E. Doyle, "Exclusive sharing and the virtualization of the cellular network," in IEEE Symposium on New Frontiers of Dynamic Spectrum Access Networks (DySPAN), Aachen Germany, 2011.

[7] C. Caicedo and M. B. Weiss, "The Viability of Spectrum Trading Markets," IEEE Communications Magazine, vol. 49, pp. 46-52, 2011.

[8] K. Arshad and K. Moessner, "Statistical Models of Spectrum Opportunities for Cognitive Radio," presented at the PIMRC, Toronto, 2011.

[9] M. B. Weiss and W. H. Lehr, "Market based approaches for dynamic spectrum assignment," Pittsburgh PA, Working Paper http://dscholarship.pitt.edu/2824/, 2009.

[10] T. W. Hazlett, "The Rationality of U.S. Regulation of the Broadcast Spectrum," The Journal of Law and Economics, vol. 33, pp. 132-175, 1990.

[11] M. B. Weiss, et al., "Sensing as a Service: An exploration into the practical implementation of DSA," in IEEE DySPAN, Singapore, 2010.

[12] M. B. H. Weiss and K. C. Huang, "To Be or Not to Be: A Comparative Study of City-wide Municipal WiFi in the US," presented at the Telecommunications Policy Research Conference, Arlington VA, 2005. 
[13] W. H. Lehr and J. M. Chapin, "Mobile Broadband Growth, Spectrum Scarcity and Sustainable Competition," presented at the Telecommunications Policy Research Conference, Arlington VA, 2011.

[14] J. E. Smith and R. F. Nau, "Valuing Risky Projects: Option Pricing Theory and Decision Analysis," Management Science, vol. 41, pp. 795-816, 1995.

[15] M. Benaroch, "Option-Based Management of Technology Investment Risk," IEEE Transactions on Engineering Management, vol. 48, pp. 428-444, 2001.
[16] J. W. Mayo and S. Wallsten, "Enabling efficient wireless communications: The role of secondary spectrum markets," Information Economics and Policy, vol. 22, pp. 61-72, Mar 2010.

[17] L. Duan, et al., "Investment and Pricing with Spectrum Uncertainty: A Cognitive Operator's Perspective," IEEE Transactions on Mobile Computing, vol. 10, pp. 1590-1604, 2011. 